\title{
The effect of the longitudinal polarization component in multi-axial nulling interferometry for exoplanet detection
}

\author{
J. F. P. Spronck and S. F. Pereira
}

\begin{abstract}
Optics Research Group, Dept. of Imaging Science and Technology, Faculty of Applied Sciences, Delft University of Technology, Lorentzweg 1, 2628 CJ Delft, The Netherlands

e-mail: julspronck@gmail.com
\end{abstract}

Received 5 March 2008 / Accepted 3 February 2009

ABSTRACT

\begin{abstract}
We show a fundamental limitation of multi-axial beam combiners in nulling interferometry. The longitudinal electric field induced by the focusing optics can drastically limit the performance of such a nulling interferometer. We further analyze the filtering capabilities of a single-mode optical fiber with respect to that longitudinal field.
\end{abstract}

Key words. technique: interferometric - instrumentation: interferometers

\section{Introduction}

In the past decade, exoplanet detection has drawn a lot of attention. Since the discovery of the first exoplanet by Mayor \& Queloz (1995), more than two hundred planets have been detected. However, direct detection of Earth-like exoplanets remains very challenging, mainly because of the huge brightness contrast between the star and the planet $\left(10^{6}\right.$ at $10 \mu \mathrm{m}$ and significantly higher in the visible) and their small angular separation (typically 0.1 arcsec). To meet this challenge, Bracewell proposed a technique in 1978 called nulling interferometry (Bracewell 1978).

This promising technique consists in observing a star-planet system with an array of telescopes (two telescopes in the original Bracewell configuration), and then combining the light from these telescopes in such a way that destructive interference occurs for the star light and simultaneously, (partially) constructive interference for the planet light. To detect a terrestrial planet, the ratio between the intensities corresponding to constructive and destructive interferences, the so-called rejection ratio, should be at least $10^{6}$. A major difficulty is that this rejection ratio should be achieved in a wide spectral band (typically from $6-18 \mu \mathrm{m}$ or even wider, Angel et al. 1986). Indeed, this wide band is required to obtain spectral information from the planet and to optimally exploit its photon flux.

To create interference, light from different telescopes must be combined in a beam combiner. With conventional optics, there are two types of beam combination: uni-axial (Serabyn \& Colavita 2001) and multi-axial (Haguenauer \& Serabyn 2006; Buisset et al. 2006) combination (see Fig. 1). In a uni-axial combiner, beams are superimposed with beam-splitters to form only one beam, which is then directed to the detector. In a multi-axial combiner, the non-superimposed beams are imaged with a focusing optics and overlap only in the image plane, where detection takes place. The advantage of the second method is that combination could be implemented with mirrors only and can therefore be achromatic. Also, this combination scheme is easily generalized to any number of beams. Unfortunately, depending on the configuration, a longitudinal component of the electric field, also referred to as longitudinal polarization, will be introduced by the focusing optics and will limit the performances of the nulling interferometer.

In this paper, we show the theoretical limitations of a multi-axial nulling interferometer with respect to longitudinal polarization. In Sect. 2, we use a simple approach to illustrate and quantify the longitudinal polarization issue. In Sect. 3, we use rigorous diffraction theory to validate the results obtained in Sect. 2. In Sect. 4, we analyze the filtering capabilities of a single-mode fiber with respect to that issue. In Sect. 5, we study the sensitivity of multi-axial nulling interferometers with respect to some imperfections. In Sect. 6, we investigate the case of nulling interferometers based on rotation of the polarization instead of phase shifting. Our conclusions are then summarized in Sect. 7.

\section{Ray tracing}

In this section, we use a simple ray tracing approach to illustrate and quantify the longitudinal polarization problem in a multi-axial beam combiner.

Consider two linearly polarized beams out of phase with equal amplitudes. We furthermore assume the beams to be monochromatic since the problem treated here does not depend on the width of the spectral band. Using a simple ray tracing model (Quabis et al. 2001), we can show that the focusing optics rotates the wave vectors $\left(k_{1}\right.$ and $\left.k_{2}\right)$ and therefore the vibration planes of the two beams (see Fig. 2a). Depending on the initial polarization, a longitudinal field is created at focus. Indeed if the beams are linearly polarized along the baseline ( $x$-polarized) and are out-of-phase, we can see in Fig. $2 \mathrm{a}$ that the resulting vector at focus is non-zero 


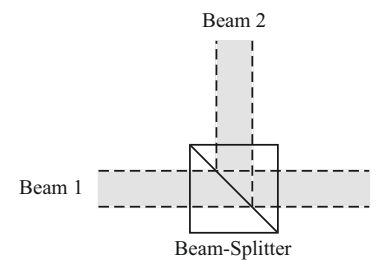

(a)

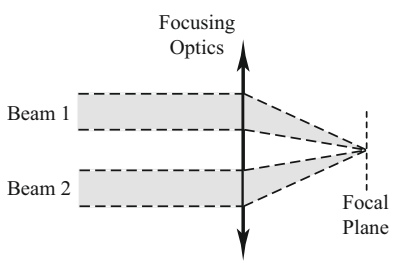

(b)

Fig. 1. a) Uni-axial and b) multi-axial combination.

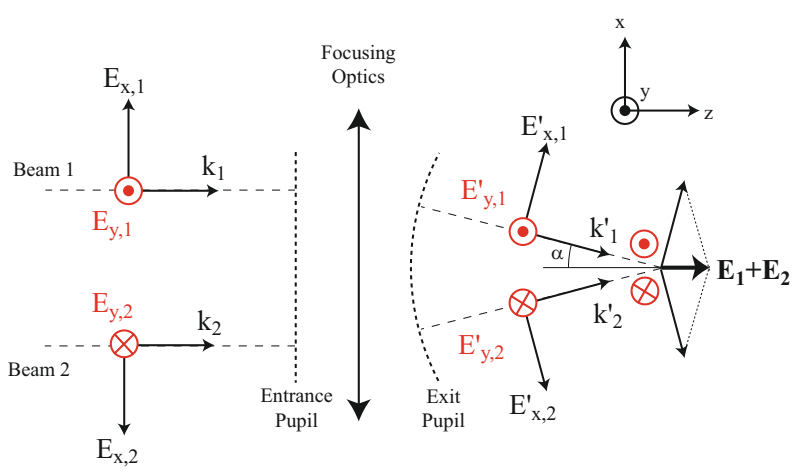

(a)

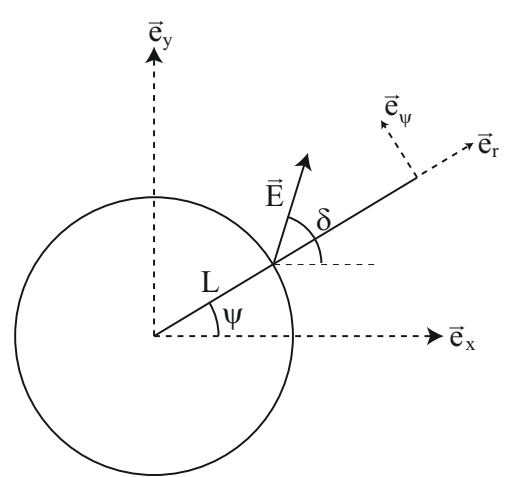

(b)

Fig. 2. a) Schematic combination of two out-of-phase linearly polarized beams. Depending on the initial orientation of the polarization, the focusing optics introduces a longitudinal component of the electric field. b) Entrance pupil of the focusing optics with a beam whose position is $(L, \psi)$ in polar coordinates and whose linear polarization is oriented at an angle $\delta$.

and purely longitudinal (z-direction). This non-zero longitudinal field will be detected and will limit the rejection ratio and therefore the performance of the nulling interferometer. If the initial polarization is perpendicular to the baseline ( $y$-polarized), the electric field remains transversal and the resulting on-axis energy density is zero. In this case, the rejection ratio is theoretically infinite.

Consider now an infinitely small beam of which the position in the entrance pupil is given in polar coordinates by $(L, \psi)($ see Fig. 2b). We can define an "effective numerical aperture" $(\sin \alpha)$ as the ratio between the semi-baseline $L$ and the focal length of the focusing optics. If the beam is linearly polarized at an angle $\delta$, the corresponding electric field in the entrance pupil $\boldsymbol{E}_{\text {ent }}$ can be written

$\boldsymbol{E}_{\mathrm{ent}}=E_{0} \cos (\delta-\psi) \boldsymbol{e}_{\mathrm{r}}+E_{0} \sin (\delta-\psi) \boldsymbol{e}_{\psi}$,

where $\boldsymbol{e}_{\mathrm{r}}$ and $\boldsymbol{e}_{\psi}$ represent the polar and azimuthal axes. The electric field in the exit pupil $\boldsymbol{E}_{\mathrm{ex}}$ is found by rotating the field $\boldsymbol{E}_{\mathrm{ent}}$ of an angle $\alpha$ around the azimuthal axis,

$$
\begin{gathered}
\boldsymbol{E}_{\mathrm{ex}}=E_{0} \cos (\delta-\psi) \cos \alpha \cdot \boldsymbol{e}_{\mathrm{r}}+E_{0} \sin (\delta-\psi) . \boldsymbol{e}_{\psi} \\
+E_{0} \cos (\delta-\psi) \sin \alpha \cdot \boldsymbol{e}_{z},
\end{gathered}
$$

which after simple trigonometric manipulations can be re-written in cartesian coordinates

$$
\begin{aligned}
\boldsymbol{E}_{\mathrm{ex}}=E_{0} & \left\{\cos \delta+\left[\cos \delta \cos ^{2} \psi+1 / 2 \sin \delta \sin (2 \psi)\right](\cos \alpha-1)\right\} \boldsymbol{e}_{x} \\
& +E_{0}\left\{\sin \delta+\left[1 / 2 \cos \delta \sin (2 \psi)+\sin \delta \sin ^{2} \psi\right](\cos \alpha-1)\right\} \boldsymbol{e}_{y} \\
& +E_{0}(\cos \delta \cos \psi+\sin \delta \sin \psi) \sin \alpha \boldsymbol{e}_{z}
\end{aligned}
$$

The field at focus $\boldsymbol{E}$ is simply given by adding the fields in the exit pupil corresponding to all individual beams $\boldsymbol{E}_{\mathrm{ex}, \mathrm{j}}$,

$\boldsymbol{E}=\sum_{j=1}^{N} \boldsymbol{E}_{\mathrm{ex}, j}$

Consider $N$ beams regularly spaced on a circle $(N>1)$. The position of the $j$ th beam in polar coordinates is given by $\left(L, \psi_{j}=\frac{2 \pi}{N}(j-1)\right), j=1, . ., N$. The beams are all linearly-polarized at an angle $\delta$ and, in order to get on-axis destructive 
interference, the phase of the $j$ th beam is chosen to be $\phi_{j}=\frac{2 \pi}{N}(j-1)$. In good approximation, the electric field at focus $\boldsymbol{E}$ is purely longitudinal and we have

$$
\begin{aligned}
& E_{z}=E_{0} \sin \alpha \cos \delta \underbrace{\sum_{j=1}^{N} \cos \left(\frac{2 \pi}{N}(j-1)\right) \exp \left(i \frac{2 \pi}{N}(j-1)\right)} \\
& \begin{cases}2 & \text { if } N=2 \\
N / 2 & \text { if } N \neq 2\end{cases} \\
& +E_{0} \sin \alpha \sin \delta \underbrace{\sum_{j=1}^{N} \sin \left(\frac{2 \pi}{N}(j-1)\right) \exp \left(i \frac{2 \pi}{N}(j-1)\right)} \\
& = \begin{cases}2 E_{0} \sin \alpha \cos \delta, & \text { if } N=2, \\
E_{0} N \sin \alpha \exp (i \delta) / 2, & \text { if } N \neq 2 .\end{cases}
\end{aligned}
$$

In order to calculate the rejection ratio, we also need the electric field in the case of a constructive interference. This purely transverse field can be calculated by setting the phase of each beam to zero. The field corresponding to constructive interference is given by

$$
\begin{aligned}
& E_{x}=E_{0} \sum_{j=1}^{N} \cos \delta+E_{0} \cos \delta \underbrace{\sum_{j=1}^{N} \cos ^{2}\left[\frac{2 \pi}{N}(j-1)\right]}_{\text {if } N=2}(\cos \alpha-1)+E_{0} / 2 \sin \delta \underbrace{\sum_{j=1}^{N} \sin \left[2 \frac{2 \pi}{N}(j-1)\right]}_{0}(\cos \alpha-1) \\
& \{N / 2, \quad \text { if } N \neq 2, \\
& = \begin{cases}E_{0} 2 \cos \delta \cos \alpha, & \text { if } N=2, \\
E_{0} N \cos \delta(\cos \alpha+1) / 2, & \text { if } N \neq 2,\end{cases}
\end{aligned}
$$

and

$$
\begin{aligned}
E_{y} & =E_{0} \sum_{j=1}^{N} \sin \delta+E_{0} / 2 \cos \delta \underbrace{\sum_{j=1}^{N} \sin \left[2 \frac{2 \pi}{N}(j-1)\right]}_{0}(\cos \alpha-1)+E_{0} \sin \delta \underbrace{\sum_{j=1}^{N} \sin ^{2}\left[\frac{2 \pi}{N}(j-1)\right]}_{ \begin{cases}0, & \text { if } N=2, \\
N / 2, & \text { if } N \neq 2,\end{cases} }(\cos \alpha-1)
\end{aligned}
$$

The electric energy density $\mathcal{U}_{+}$corresponding to constructive interference is then given, within a constant factor, by

$\mathcal{U}_{+}= \begin{cases}4\left|E_{0}\right|^{2}\left(\cos ^{2} \delta \cos ^{2} \alpha+\sin ^{2} \delta\right), & \text { if } N=2 \\ \left|E_{0}\right|^{2} N^{2}(\cos \alpha+1)^{2} / 4, & \text { if } N \neq 2\end{cases}$

which shows as expected that the power corresponding to constructive interference is proportional to $N^{2}$.

The rejection ratio is found by dividing the electric energy densities corresponding to constructive and destructive interferences,

$R= \begin{cases}\frac{\left(\cos ^{2} \delta \cos ^{2} \alpha+\sin ^{2} \delta\right)}{\sin ^{2} \alpha \cos ^{2} \delta}, & \text { if } N=2, \\ \frac{(\cos \alpha+1)^{2}}{\sin ^{2} \alpha}, & \text { if } N \neq 2 .\end{cases}$

This expression can be simplified in the case of very small effective numerical apertures ( $\cos \alpha \approx 1$ ), leading to

$R \approx \begin{cases}\frac{1}{\sin ^{2} \alpha \cos ^{2} \delta}, & \text { if } N=2, \\ \frac{4}{\sin ^{2} \alpha}, & \text { if } N \neq 2 .\end{cases}$

From Eq. (10), we can see that the rejection ratio is inversely proportional to the square of the effective numerical aperture $(\sin \alpha)$. We can also see that when $N>2$, the rejection ratio does not depend on the orientation of the incoming linear polarization. In the two-beam case, as we discussed previously, the rejection ratio is infinite if the polarization is perpendicular to the baseline $(\delta=\pi / 2)$ and minimum if the polarization is along the baseline $(\delta=0)$. Note that for small effective numerical apertures, the electric energy density $\mathcal{U}_{+}$corresponding to constructive interference is $\mathcal{U}_{+}=\left|E_{0}\right|^{2} N^{2}$, which is the expected value for the constructive interference of an $N$-beam interferometer. 


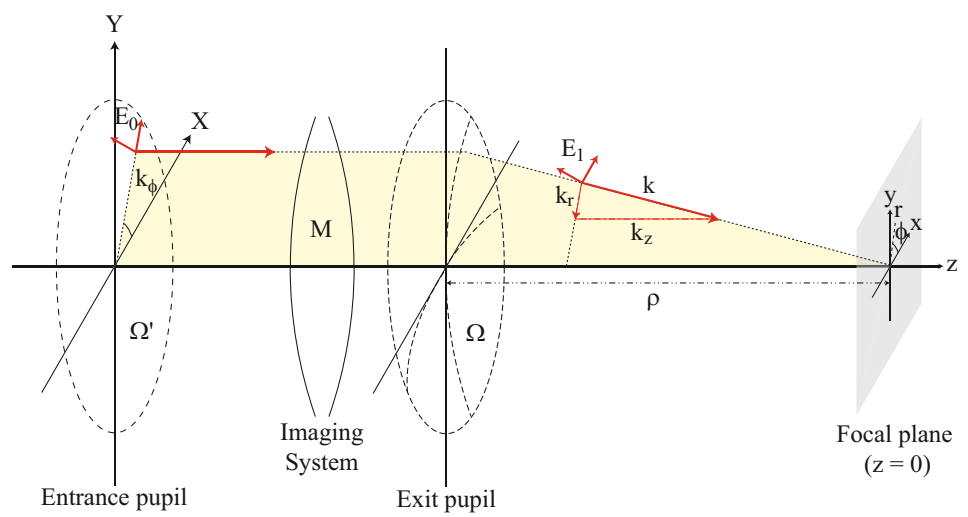

Fig. 3. Schematic overview of the studied configuration. Light distribution in the entrance pupil $\boldsymbol{E}_{0}\left(k_{\mathrm{r}}, k_{\phi}, 0\right)$ is mapped to the exit pupil $\boldsymbol{E}_{1}\left(k_{\mathrm{r}}, k_{\phi}, k_{z}\right)$ via an aplanatic imaging system, denoted by the operation $\boldsymbol{M}$. The field distribution in the focal region is then obtained by integration over the exit pupil. The focal plane is the plane $z=0$.

\section{Electric field distribution}

In Sect. 2, we showed, based on geometrical considerations, that longitudinal polarization will limit the rejection ratio of a multiaxial nulling interferometer. In order to validate these results in the case of extended beams, we will, in this section, perform an analysis of the three-dimensional electric field distribution in the focal plane of the focusing optics using rigorous diffraction theory.

\subsection{Theory}

Let us consider the aplanatic imaging system depicted in Fig. 3. In this imaging system, the electric field in the entrance pupil $\boldsymbol{E}_{0}\left(k_{\mathrm{r}}, k_{\phi}, 0\right)$ is mapped to the exit pupil $\boldsymbol{E}_{1}\left(k_{\mathrm{r}}, k_{\phi}, k_{z}\right)$ according to the aplanatic condition. This exit pupil is a spherical shell with radius $\rho$. To describe the electric field, we introduce two sets of cylindrical coordinates: $\boldsymbol{k}=\left(k_{\mathrm{r}}, k_{\phi}, k_{z}\right)$ in the exit pupil and $\boldsymbol{r}=(r, \phi, z)$ in the focal region, where the plane $z=0$ is the focal plane. If we consider a monochromatic time-harmonic electric field, we can calculate the electric field distribution in the focal plane using diffraction integrals described by Ignatowsky (1919) and re-derived by Richards \& Wolf (1959). These diffraction integrals are valid in the Debye approximation and therefore our point of observation should not be too close to the spherical shell $\Omega$ over which the integration takes place. Nevertheless, it is more convenient to integrate over the entrance pupil $\Omega^{\prime}$ rather than over the exit pupil $\Omega$. In a weakly-aberrated and aplanatic imaging system with the object at infinity, the transition from the entrance pupil to the exit pupil can be considered as a rotation of the wave vector, described by the propagation matrix $\boldsymbol{M}$ (van de Nes et al. 2004)

$\boldsymbol{M}=\frac{1}{k}\left(\begin{array}{cc}k_{z} \cos ^{2} k_{\phi}+k \sin ^{2} k_{\phi} & \left(k_{z}-k\right) \cos k_{\phi} \sin k_{\phi} \\ \left(k_{z}-k\right) \cos k_{\phi} \sin k_{\phi} & k_{z} \sin ^{2} k_{\phi}+k \cos ^{2} k_{\phi} \\ -k_{\mathrm{r}} \cos k_{\phi} & -k_{\mathrm{r}} \sin k_{\phi}\end{array}\right)$,

where $k=2 \pi / \lambda$ is the wave number. This matrix is a $(3 \times 2)$-matrix since, with the foregoing assumptions, the electric field has no $z$-component in the entrance pupil.

Taking these considerations into account, the electric field in the focal plane is given by (van de Nes et al. 2004)

$\boldsymbol{E}(r, \phi, 0)=-\frac{i \rho}{2 \pi} \iint_{\Omega^{\prime}} \sqrt{\frac{k_{z}}{k}} \frac{\boldsymbol{M} \cdot \boldsymbol{E}_{0}\left(k_{\mathrm{r}}, k_{\phi}\right)}{k_{z}} \exp \left[i r k_{\mathrm{r}} \cos \left(k_{\phi}-\phi\right)\right] k_{\mathrm{r}} \mathrm{d} k_{\mathrm{r}} \mathrm{d} k_{\phi}$.

This expression allows us to calculate the three-dimensional distribution of the electric field in the focal plane $\boldsymbol{E}(r, \phi, 0)$ given a certain field $\boldsymbol{E}_{0}\left(k_{\mathrm{r}}, k_{\phi}\right)$ in the entrance pupil. The electric energy density in the focal plane is then given by

$\mathcal{U}(r, \phi)=\frac{\epsilon_{0}}{2}\left[\left|E_{x}(r, \phi)\right|^{2}+\left|E_{y}(r, \phi)\right|^{2}+\left|E_{z}(r, \phi)\right|^{2}\right]$

To calculate the rejection ratio $R$, we need the on-axis densities corresponding to constructive $\left(\mathcal{U}_{+}\right)$and destructive $\left(\mathcal{U}_{-}\right)$interferences.

$R=\frac{\mathcal{U}_{+}(r=0)}{\mathcal{U}_{-}(r=0)}$

Note that Eq. (14) only gives a theoretical rejection ratio since in practice, one would need an infinitely small, mono-pixel detector. In reality, the detector has a certain extent and will therefore capture more light. Therefore, the rejection ratio as defined in Eq. (14) gives a maximal theoretical limit. Note also that the case of a pinhole-detector is not taken into account here, since a realistic pinhole size will automatically lead to a very poor performance of the multi-axial nulling interferometer. 


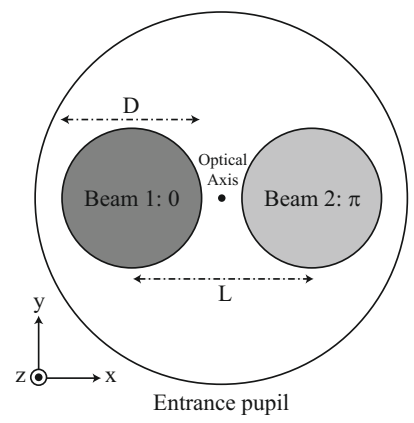

(a)

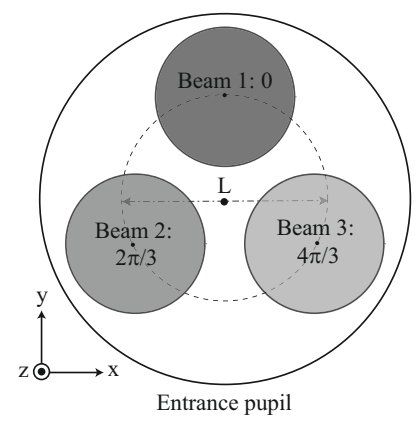

(b)

Fig. 4. Schematic entrance pupil for the combination of a) two beams positioned along the $x$-axis and b) three beams. There is a phase-shift between the beams in order to have on-axis destructive interference. The applied phases are a) 0 and $\pi$ and $\mathbf{b}) 0,2 \pi / 3$ and $4 \pi / 3$. The diameter of the beams is $D$ and the baseline is $L$.

\subsection{Simulations}

Nulling interferometry can be performed with any number $N$ of beams. However, in practice, a small number of beams $(N \leq 3)$ is generally proposed. Therefore, in this section, we will only consider two- and three-beam nulling interferometers.

We use in all simulations linearly polarized beams with equal and constant amplitudes (top-hat distribution) and the phase of the $j$ th beam is chosen to be $\phi_{j}=\frac{2 \pi}{N}(j-1)$ in order to get on-axis destructive interference. The diameter of the beams is $D=2 \mathrm{~cm}$ and the wavelength is $600 \mathrm{~nm}$. The focusing optics that we simulated has a focal length $f=60 \mathrm{~cm}$ and the distance between the beams in the entrance pupil, called the baseline, is $L=5 \mathrm{~cm}$. Note that for a number of beams $N>2$, we can define the baseline as the diameter of the circle on which the centers of the beams are positioned. For $N=2$, these two definitions coincide. The values of the different parameters have been chosen to match our table-top experimental set-up, the Delft Testbed Interferometer (DTI). It is a three-beam multi-axial interferometer, initially designed for homothetic mapping (Gori et al. 2004).

\section{Two-beam interference}

In this case, we consider multi-axial combination of two beams (see Fig. 4a). The baseline is along the $x$-axis and we consider linearly polarized beams along the $x$ - and $y$-axis.

The amplitudes of the three-dimensional electric field components in the focal plane for $x$ - and $y$-polarized beams are respectively depicted in Figs. 5a and 5b. In each case, the three components of the field have been normalized to the largest component $\left(E_{x}\right.$ for $x$-polarization and $E_{y}$ for $y$-polarization). In both cases, the main field component shows the well-known interference fringes and the other transverse component is negligible. Only the modulus of the field components is depicted in Fig. 5a and Fig. 5b. If the phase was also represented, we would see that the longitudinal components are centro-symmetric while the transverse components are anti-symmetric and therefore equal to zero on-axis ( $x=0$ and $y=0)$ whatever the polarization. When the polarization is perpendicular to the baseline (see Fig. 5b), the on-axis longitudinal field is also equal to zero in such a way that the on-axis energy density is null. The rejection ratio is therefore infinite. As expected from the ray-tracing model, when the polarization is along the baseline (see Fig. 5a), the on-axis longitudinal component is no longer equal to zero, implying a limited rejection ratio. In this case, the on-axis longitudinal component is equal to 0.042 . Because of normalization, this gives a rejection ratio of $R=1 / 0.042^{2}=575$.

An experimental verification is possible if one uses a detection system with high spatial resolution. Indeed, in the considered geometrical configuration, the size of the fringes is of the order of $7 \mu \mathrm{m}$. The rejection ratio is limited due to the finite size of the pixels. To be able to detect the limitation of the rejection ratio by the longitudinal component, one would need a pixel size of the order of $100 \mathrm{~nm}$, which is much smaller than the pixel of any CCD camera. To reduce the spatial resolution issue, one could increase the size of the fringes by using a larger focal length or a shorter baseline. Unfortunately, as we will see in the last part of this section, this would lead to a higher rejection ratio, which requires a smaller pixel size (see end of this section for details). Therefore possible ways to overcome this issue are the use of a high resolution recording media, such as a photoresist (Hao \& Leger 2007), or a polarization-sensitive point detector such as single molecules (Novotny et al. 2001) or quantum well heterostructures (Rurimo et al. 2006).

\section{Three-beam interference}

In this case, we consider the combination of three beams regularly spaced on a circle of diameter $L=5 \mathrm{~cm}$ as depicted in Fig. $4 \mathrm{~b}$. In order to have on-axis destructive interference, the phases of the different beams have been respectively chosen to be equal to 0 , $2 \pi / 3$ and $4 \pi / 3$.

The field distributions for $x$ - and $y$-polarizations are depicted in Figs. 5c and 5d. In the three-beam case, none of the components are either symmetric or anti-symmetric and the on-axis longitudinal field is always non-zero. The rejection ratio of such a threebeam multi-axial nulling interferometer is therefore limited for both $x$ - and $y$-polarizations. The on-axis longitudinal component is equal to 0.021 , which gives a rejection ratio of $R=1 / 0.021^{2}=2300$. 

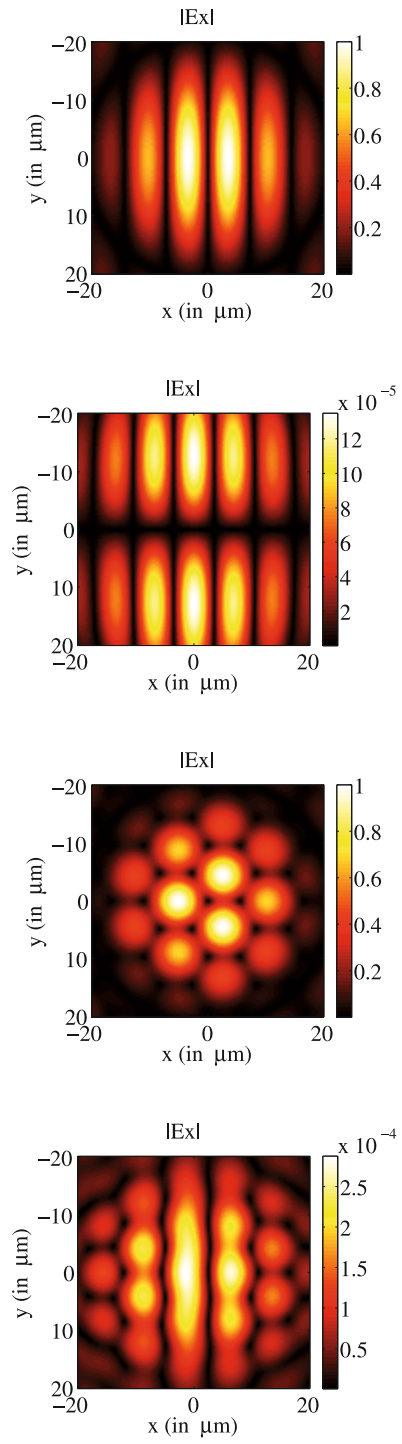

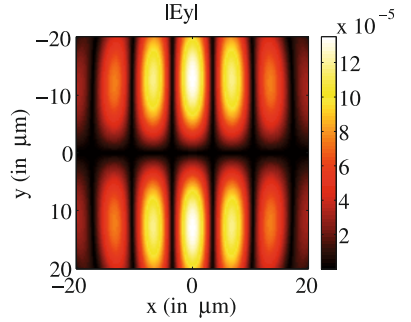

(a)

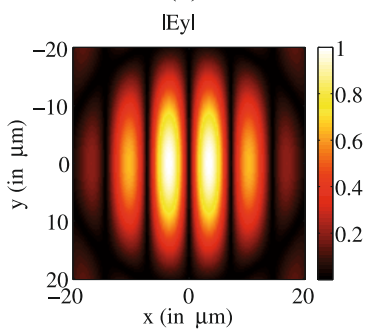

(b)

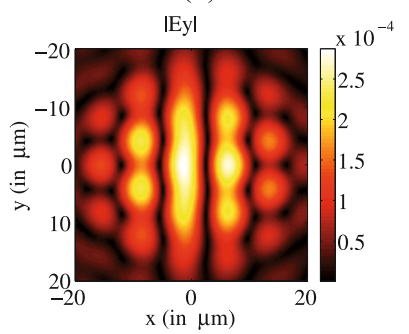

(c)

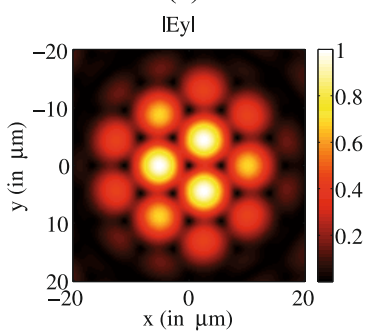

(d)
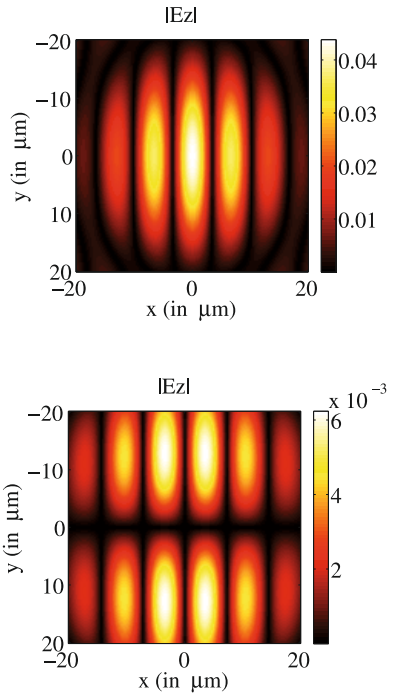

|Ez|

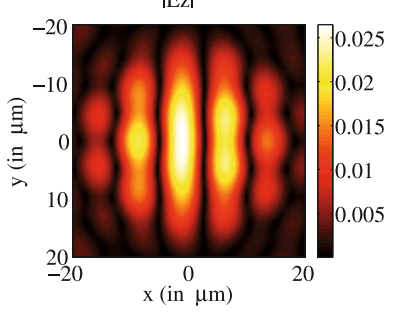

$\mid$ Ez $\mid$

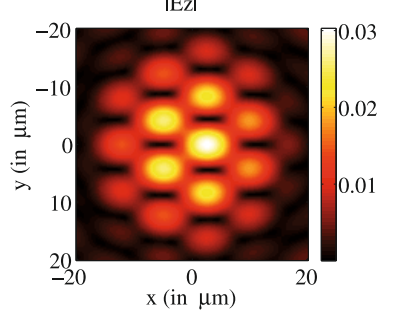

Fig. 5. Three components of the electric field $\left(\left|E_{x}\right|,\left|E_{y}\right|\right.$ and $\left.\left|E_{z}\right|\right)$ in the focal plane of the focusing optics in the case of (a) and b)) two-beam and (c) and d)) three-beam multi-axial combiner. The beams are linearly polarized along either the $x$-axis (a) and c)) or the $y$-axis (b) and d)). In each case, the three components of the field have been normalized to the largest component ( $E_{x}$ for $x$-polarization and $E_{y}$ for $y$-polarization).

\section{$N$-beam interference}

As shown by Eq. (10), the rejection ratio in the case of a $N$-beam multi-axial interferometer is limited for both polarizations to the same value for all $N>2$.

\section{Influence of the numerical aperture}

As mentioned in Sect. 2, we define the effective numerical aperture $\left(N A_{\mathrm{eff}}\right)$ as the ratio between the semi-baseline $L / 2$ and the focal length of the focusing optics $f$,

$N A_{\mathrm{eff}}=\frac{L}{2 f}$.

The rejection ratio as a function of the effective numerical aperture in the case of two and three beams is depicted in Fig. 6 . The dots represent the rejection ratio calculated with rigorous diffraction theory, while the lines have been calculated using Eq. (10). We see that for both two- and three-beam cases, the agreement between ray tracing and diffraction theory is very good and that the rejection ratio is inversely proportional to the square of the effective numerical aperture.

As discussed in the beginning of this section, decreasing the effective numerical aperture does not solve the spatial resolution issue. Indeed, imagine that in order to increase the interfringe, we divide the effective numerical aperture by a factor of two. This implies that one can use a pixel twice as large to detect the same rejection ratio. However, the longitudinal component will also be twice as small, which means that the rejection ratio that we would like to achieve should be four times higher. Since the rejection 


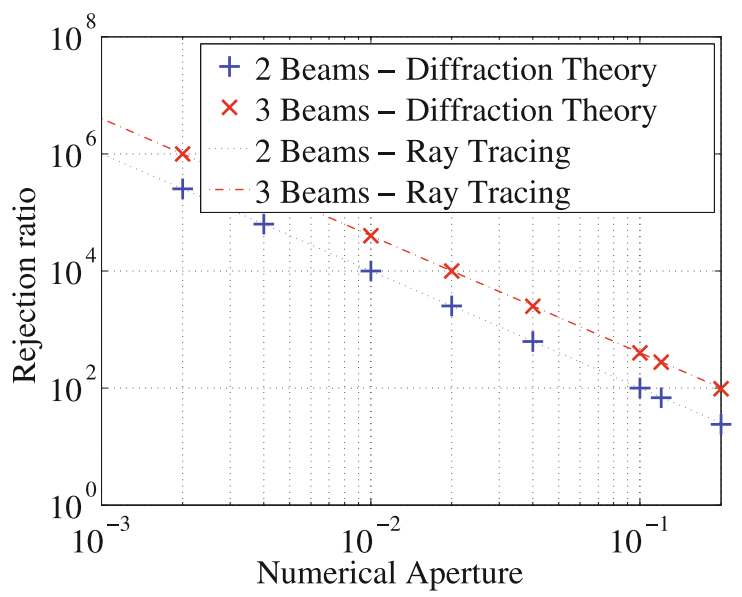

Fig. 6. Rejection ratio as a function of the effective numerical aperture.

ratio is also inversely proportional to the square of the pixel size, a four times higher rejection ratio implies a pixel twice as small. The optimal pixel size is therefore independent of the effective numerical aperture.

In a real nulling interferometer, the effective numerical aperture is typically 0.06 in order to match the acceptance angle of the single-mode fiber (used for wavefront filtering). The corresponding rejection ratio is of the order of $10^{2}$ for two beams and $10^{3}$ for three beams. This is definitely too low for Earth-like exoplanet detection and such a multi-axial nulling interferometer therefore cannot be used as such. A solution needs to be found to solve this fundamental problem.

\section{Longitudinal polarization and single-mode fibers}

In Sect. 3, we studied the electric field distribution in the focal plane of the focusing optics and we concluded that the rejection ratio of a multi-axial nulling interferometer can be drastically limited due to longitudinal polarization. However, a wavefront filter is needed to have quasi-perfect destructive interference. This filter will affect the field distribution and therefore the rejection ratio. In the case of multi-axial nulling interferometry, an efficient way to perform wavefront filtering is to focus the light from the different beams into a unique single-mode fiber (positioned at the focus of the imaging system) (Mennesson et al. 2002; Wallner et al. 2004). In this section, we will study the filtering capabilities of a single-mode fiber with respect to the longitudinal polarization issue. We will consider perfectly lossless and perfectly single-mode fibers. In reality, these fibers are not ideal and the lack of single-modeness might limit the performance of the interferometer. However, these issues are out of the scope of this paper.

\subsection{Theory}

If we neglect losses inside the fiber, the output field of an optical fiber can be described in terms of complex coupling efficiencies (Mennesson et al. 2002; Wallner \& Leeb 2002; Wallner et al. 2003). The coupling efficiency $\xi_{j}$ to a certain mode $\boldsymbol{E}_{j}(x, y)$ is the (complex) strength with which an incoming field focused onto the optical fiber will excite this mode. A single-mode fiber has two orthogonal fundamental modes corresponding to the two orthogonal polarizations. In order to keep the same nomenclature as in Snyder \& Love (1983), we will call these modes even $\boldsymbol{E}_{\mathrm{e}}$ and odd $\boldsymbol{E}_{\mathrm{o}}$. An expression for these modes is given in Snyder \& Love (1983). The electric field at the output of the fiber is then given by

$\boldsymbol{E}(x, y)=\xi_{\mathrm{e}} \boldsymbol{E}_{\mathrm{e}}(x, y)+\xi_{\mathrm{o}} \boldsymbol{E}_{\mathrm{o}}(x, y)$,

where $\xi_{\mathrm{e}}$ and $\xi_{\mathrm{o}}$ are the complex coupling efficiencies corresponding to these modes. An expression for these coupling efficiencies is given in Appendix A.

As shown in Appendix A, the rejection ratio after fiber filtering is given by

$R_{\text {out }}=\frac{\left|\xi_{\mathrm{e},+}\right|^{2}+\left|\xi_{\mathrm{o},+}\right|^{2}}{\left|\xi_{\mathrm{e},-}\right|^{2}+\left|\xi_{\mathrm{o},-}\right|^{2}}$,

where the subscript + denotes coupling efficiencies in the case of constructive intereference and - corresponds to the case of destructive interference. Note that this expression is only valid in the monochromatic case.

An important remark to mention about the coupling efficiencies of a single-mode fiber is that, because of the central symmetry of the fundamental modes (see Appendix A), an incoming electric field with anti-symmetric transverse components will not be coupled into the fiber, since both coupling efficiencies $\xi_{\mathrm{e}}$ and $\xi_{\mathrm{o}}$ would be equal to zero in this case.

Consider $N$ ray-like beams regularly spaced on a circle. The position of the $j$ th beam in polar coordinates is given by $\left(k_{\mathrm{r}}, k_{\phi, j}=\frac{2 \pi}{N}(j-1)\right), j=1, \ldots, N$. The beams are linearly-polarized at an angle $\delta$ and, in order to get on-axis destructive interference, the phase of the $j$ th beam is chosen to be $\phi_{j}=\frac{2 \pi}{N}(j-1)$. As presented in Appendix B, the coupling efficiencies to the odd 
mode $\xi_{\mathrm{o},-}$ and to the even mode $\xi_{\mathrm{e},-}$ are respectively given by

$\xi_{\mathrm{o},-}=\left\{\begin{array}{ll}-3 i \pi K \exp (-i \delta)\left(G_{1} I_{2}+G_{2} I_{1}\right), & \text { if } N=3 \\ 0, & \text { if } N \neq 3\end{array}\right.$,
$\xi_{\mathrm{e},-}=\left\{\begin{array}{ll}3 \pi K \exp (-i \delta)\left(G_{1} I_{2}+G_{2} I_{1}\right), & \text { if } N=3 \\ 0, & \text { if } N \neq 3\end{array}\right.$,

where $I_{1}=\int_{0}^{\infty} J_{0}\left(r k_{\mathrm{r}}\right) A_{1}(r) r \mathrm{~d} r, I_{2}=\int_{0}^{\infty} J_{2}\left(r k_{\mathrm{r}}\right) A_{2}(r) r \mathrm{~d} r, J_{0}$ and $J_{2}$ denote respectively the zeroth and the second order Bessel functions of the first kind and $K, G_{1}, G_{2}$ are defined in Appendix B.

In order to calculate the rejection ratio, we need to know the coupling efficiencies corresponding to the constructive interference. In this case, the beams are all in phase $\left(\phi_{j}=0\right)$. After calculations, we find (for $N \neq 2$ )

$\xi_{\mathrm{o},+}=-2 \pi K N \sin (\delta)\left(G_{1} I_{1}+G_{2} I_{2}\right)$,

$\xi_{\mathrm{e},+}=-2 \pi K N \cos (\delta)\left(G_{1} I_{1}+G_{2} I_{2}\right)$.

In the case of constructive interference, the detected power would be given by

$P_{\text {out }}=4 \pi^{2} K^{2} N^{2}\left|G_{1} I_{1}+G_{2} I_{2}\right|^{2} P$,

which shows that the photon flux coming from the planet is proportional to $N^{2}$.

The rejection ratio is then found using Eq. (17):

$R_{\text {out }}=\left\{\begin{array}{ll}2\left|\frac{G_{1} I_{1}+G_{2} I_{2}}{G_{1} I_{2}+G_{2} I_{1}}\right|^{2}, & \text { if } N=3 \\ \infty, & \text { if } N \neq 3\end{array}\right.$.

For very small effective numerical apertures $\left(N A_{\text {eff }}<0.01\right)$, we have

$G_{2} / G_{1} \propto \mathrm{NA}_{\text {eff }}^{2}$ and $I_{2} / I_{1} \propto \mathrm{NA}_{\text {eff }}^{2}$,

in such a way that $G_{2} I_{2} / G_{1} I_{1} \propto N A_{\text {eff }}^{4}$. The rejection ratio is therefore given in good approximation by

$R_{\text {out }}=\left\{\begin{array}{ll}2\left|\frac{1}{G_{2} / G_{1}+I_{2} / I_{1}}\right|^{2} \propto N A_{\text {eff }}^{4}, & \text { if } N=3 \\ \infty, & \text { if } N \neq 3\end{array}\right.$.

Except in the case $N=3$, both coupling efficiencies $\xi_{\mathrm{o},-}$ and $\xi_{\mathrm{e},-}$ are equal to zero in such a way that no light will be coupled in the fiber. The rejection ratio will therefore be theoretically infinite for $N \neq 3$. When $N=3$, the rejection ratio is in good approximation inversely proportional to the fourth power of the effective numerical aperture while it was inversely proportional to the square of the effective numerical aperture without optical fiber. We can also see in Eq. (23) that the rejection ratio does not depend on the initial direction of the polarization. Note that the rejection ratio given in Eq. (21) is only rigorous for ray-like beams and not for finite-size beams.

\subsection{Simulations}

In this section, we perform numerical simulations in order to validate the results obtained in Sect. 4.1 and in order to extend these results to finite-size beams. In all simulations, propagation losses inside the fiber are neglected. Only the coupling losses (due to a mismatch between the incoming field and the mode of the fiber) are taken into account.

In our simulations, we use a wavelength of $600 \mathrm{~nm}$ and a step-index single-mode fiber with an acceptance angle of 0.125 and a core radius $a=1.2 \mu \mathrm{m}$. We assume a core material with a refractive index $n_{\mathrm{co}}=1.45$. For such a fiber, the two orthogonal fundamental modes are given in Snyder \& Love (1983).

Using the results of Sect. 3 for the electric field distributions in the focal plane, we can calculate the different coupling efficiencies in Eq. (A.2) and the rejection ratio in Eq. (17).

For $N \neq 3$, we indeed find an infinite rejection ratio for all incoming polarizations and effective numerical apertures. The rejection ratio as a function of the effective numerical aperture in the case $N=3$ is depicted in Fig. 7 (stars, dotted line). The dot-markers dotted line is the theoretical curve calculated with Eq. (23). We can see that the agreement between these simulations and the theoretical rejection ratio is very good for $N A_{\text {eff }}<0.01$. For these low effective numerical apertures, the rejection ratio is inversely proportional to the fourth power of the effective numerical aperture, as predicted by Eq. (23). For larger effective numerical apertures, both curves start diverging from each other but remain of the same order of magnitude. For a typical effective numerical aperture of 0.06 (half the acceptance angle of the fiber), the rejection ratio would be of the order of $10^{6}$ and would therefore be high enough for Earth-like planet detection.

In all cases, our extended-beam simulations are in very good agreement with the theoretical results obtained in Sect. 2 with ray-like beams. 


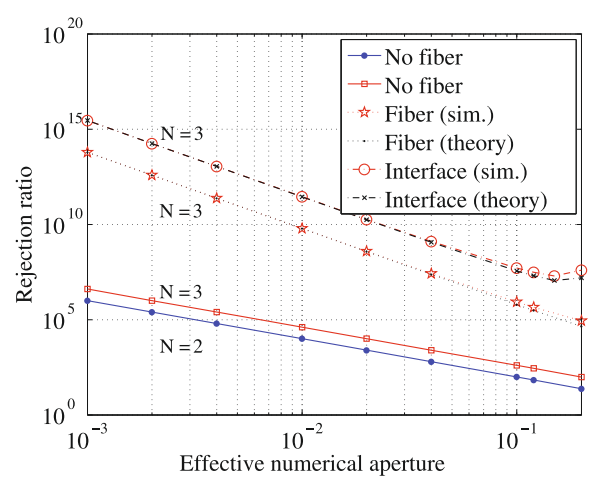

Fig. 7. Rejection ratio as a function of the effective numerical aperture in the case of two beams (dots, solid line) and three beams (squares, solid line) without fiber filtering and in the case of three beams after the fiber (stars, dotted line). The circle-markers dash-dotted line corresponds to the three-beam case after fiber filtering when Fresnel reflections are taken into account. The dot-markers dotted line and the cross-markers dash-dotted line are the theoretical curves obtained with Eq. (23).

Table 1. Rejection ratio of a three-beam multi-axial nulling interferometer when the output is coupled to a chalcogenide (mid-IR) or standard (visible) single-mode optical fiber.

\begin{tabular}{lccccc}
\hline \hline & $\lambda$ & Acceptance angle & $n_{\text {clad }}$ & $\begin{array}{c}\text { Rejection ratio } \\
\text { (No Interface) }\end{array}$ & $\begin{array}{c}\text { Rejection ratio } \\
\text { (Interface) }\end{array}$ \\
\hline Standard fiber & $600 \mathrm{~nm}$ & 0.125 & 1.45 & $4.9 \times 10^{6}$ & $1.6 \times 10^{8}$ \\
Chalcogenide fiber & $10.6 \mu \mathrm{m}$ & 0.13 & 2.927 & $4.7 \times 10^{6}$ & $1.7 \times 10^{7}$ \\
\hline
\end{tabular}

\section{Field redistribution in the fiber medium due to Fresnel reflections}

When coupled into an optical fiber, light goes through an air-glass interface. Some of the light does not go through the interface but is reflected back. This reflection, called Fresnel reflection, depends on the angle of incidence as well as the polarization of the incoming light and thus can have an effect on the rejection ratio since it affects the field distribution and therefore the coupling efficiencies with the optical fiber. In this section, we calculate the rejection ratio after fiber filtering taking into account the full vectorial model for air-glass interface.

We consider an air-glass $(n=1.45)$ interface in the focal plane of the focusing optics. Using the formalism described by van de Nes et al. (2004), which is a generalization of Eq. (12) to multilayer media, we can rigorously calculate the field distribution at a certain distance (we chose one wavelength) after the interface. The coupling efficiencies (and therefore the rejection ratio) corresponding to these field distributions can then be calculated using Eq. (A.2).

The rejection ratio for three-beam interference as a function of the effective numerical aperture is depicted in Fig. 7 (circles, dash-dotted line). We can see a very good agreement with the theoretical rejection ratio calculated with Eq. (23) (crosses, dash-dotted line). We can see that the presence of the interface improves the rejection ratio (still inversely proportional to the fourth power of the effective numerical aperture for $\mathrm{NA}_{\mathrm{eff}}<0.01$ ). In the case of a effective numerical aperture of $\mathrm{NA}_{\text {eff }}=0.06$, the rejection ratio would be of the order of $10^{8}$. Note that the rejection ratio for $N \neq 3$ is still infinite for all effective numerical apertures.

Consider now the case of single-mode chalcogenide fibers (Houizot et al. 2007). These fibers are designed to be single-mode in the mid-IR (around $10.6 \mu \mathrm{m}$ ) and are made out of high refractive index glasses. The studied fiber has a core with a refractive index of 2.927. Due to this high refractive index, such a material would highly increase the Fresnel losses. In Table 1, we can see the comparison between a chalcogenide fiber (at $10.6 \mu \mathrm{m}$ ) and a standard single-mode fiber in the visible (at $600 \mathrm{~nm}$ ). The comparison has been made at an effective numerical aperture of $\mathrm{NA}_{\mathrm{eff}}=0.06$, which corresponds to the optimal coupling efficiency in the case of the constructive interference. We see that, when the Fresnel reflection is not taken into account, both fibers lead to similar rejection ratios. However, this is not true when the air-glass interface is taken into account. With a standard visible single-mode fiber, the rejection ratio is of the order of $10^{8}$, while it is only $10^{7}$ with a chalcogenide fiber. Since the rejection ratio is improved by the presence of the interface, we expected it to increase with the refractive index of the fiber and therefore with the Fresnel losses. This result shows that the rejection ratio does not increase with the Fresnel losses but must rather be connected to the redistribution of the electric field after the interface. However, the relation between this electric field redistribution and the rejection ratio is still unclear at this moment.

Even though the interface improves the rejection ratio, it also leads to a reduction of the photon flux coming from the planet. The photon flux has been numerically estimated to be $66 \%$ of the flux obtained without interface. Therefore, a trade-off has to be made between high rejection ratio and photon flux. If a $10^{6}$ rejection ratio is sufficiently high, then an anti-reflection coating should be applied to reduce Fresnel losses and therefore to gain photons. In the case of chalcogenide fibers, the interface does not improve significantly the rejection ratio. It might therefore be important to preserve the photon flux by reducing the injection losses. 


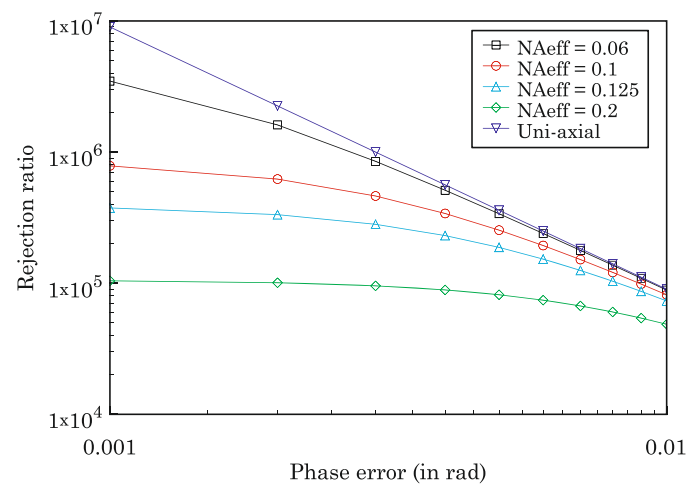

Fig. 8. Rejection ratio as a function of the phase error.

Table 2. Allowed phase errors to reach a $10^{5}$ and $10^{6}$ rejection ratio for a three-beam multi-axial nulling interferometer depending on the numerical aperture.

\begin{tabular}{ccc}
\hline \hline$N A_{\text {eff }}$ & $\delta \phi\left(10^{5}\right)$ & $\delta \phi\left(10^{6}\right)$ \\
\hline 0.06 & 0.0095 & 0.003 \\
0.1 & 0.009 & $/$ \\
0.125 & 0.008 & $/$ \\
0.2 & 0.002 & $/$ \\
\hline
\end{tabular}

\section{Consequences for the DARWIN/TPF mission}

Darwin/TPF (Fridlund 2000; Beichman et al. 1999) is a space mission aimed at Earth-like planet detection by means of nulling interferometry. Many telescope configurations have been considered for its implementation involving three to six telescopes. The latest design, the Emma X-array architecture (Lay et al. 2007; Lawson et al. 2008), is a four-telescope configuration. It consists of two pairs of telescopes, each pair acting as a separate nulling interferometer, configured in a 6:1 rectangular array (typically $120 \times 20 \mathrm{~m}$ to $400 \times 67 \mathrm{~m}$ ). The light coming from these telescopes is then sent to an out-of-plane beam combiner located typically $1 \mathrm{~km}$ away. Even though polarization issues might arise due to this out-of-plane combination, it totally depends on the optical design of the beam combiner. If well designed, the beams can still have identical polarization and lead to theoretical infinite rejection ratio. The small amount of known information makes it very difficult to estimate these effects. However, the effect is claimed to be negligible. If so, longitudinal polarization issue may still occur during the internal beam combination (which can have any numerical aperture and can even be uni-axial if desired). Consider these four perfect beams being combined with a multi-axial beam combiner. We have seen in this section that the rejection ratio of such a four-beam nulling interferometer (or of two independent pairs of two-beam nulling interferometers) would be theoretically infinite as long as the beams are regularly positioned before the final focusing optics. The last assumption is reasonable since it ensures optimal coupling efficiency with the fiber. Note that this argument would still be valid if the beams were not regularly spaced as long as the beam configuration has central symmetry (as it is the case for rectangular arrays). We can therefore assume that longitudinal polarization should not drastically affect the performance of the Darwin/TPF mission. However, special care should be taken to fully calculate the three-dimensional field induced by all encountered optics to be sure that the rejection ratio will not be limited to a non-acceptable level.

\section{Sensitivity to imperfections}

In this section, we study the influence of imperfections or misalignments on the performance of a multi-axial nulling interferometer. We only consider here the case of a three-beam interferometer and two relevant types of errors will be studied: phase and polarization mismatches.

Consider first three beams regularly spaced on a circle, as depicted in Fig. $4 \mathrm{~b}$. If the phases of the beams are respectively 0 , $2 \pi / 3$ and $4 \pi / 3$, we have seen that the rejection ratio after the fiber was limited and inversely proportional to the fourth power of the effective numerical aperture. If we introduce a phase error $\delta \phi$ in one of the beams, we expect to have a degradation of the performance of the interferometer. The rejection ratio as a function of the phase error for different effective numerical apertures $(0.06,0.1,0.125$ and 0.2$)$ is depicted in Fig. 8 . We also plotted the theoretical rejection ratio in the case of a uni-axial interferometer, which is simply given for a three-beam interferometer by $R \approx 9 / \delta \phi^{2}(\delta \phi \ll 1)$. We can see as expected that the rejection ratio decreases as the phase error increases. For decreasing numerical apertures, the rejetion ratio gets closer to the uni-axial case. We can also see that longitudinal polarization is the main source of limitation for small phase errors (of the order of $10^{-3}$ ) but when increasing, the phase error becomes dominant, independently of the type of beam combination. In other terms, multi-axial and uniaxial combiners give similar results for large phase errors (of the order of $10^{-2}$ ). We can see on Table 2 the allowed phase errors for various effective numerical apertures in order to reach a rejection ratio of $10^{5}$ and of $10^{6}$. 
Table 3. Allowed polarization errors $(\epsilon)$ to reach a $10^{5}$ and $10^{6}$ rejection ratio for a three-beam multi-axial nulling interferometer depending on the numerical aperture.

\begin{tabular}{ccc}
\hline \hline$N A_{\text {eff }}$ & $\epsilon\left(10^{5}\right)$ & $\epsilon\left(10^{6}\right)$ \\
\hline 0.06 & 0.009 & 0.002 \\
0.1 & 0.007 & $/$ \\
0.125 & 0.005 & $/$ \\
0.2 & 0.001 & $/$ \\
\hline
\end{tabular}

Consider now that the three beams have respective phases $0,2 \pi / 3$ and $4 \pi / 3$. Imperfections in the optics can induce birefringence. This birefringence will cause linear polarization to become slightly elliptical. To study this effect, we consider two perfectly linearly polarized beams and one beam with a slightly elliptical polarization,

$\boldsymbol{E}_{\mathbf{1}}=\boldsymbol{E}_{\mathbf{2}}=\left(\begin{array}{l}1 \\ 0\end{array}\right)$ and $\boldsymbol{E}_{3}=\left(\begin{array}{l}1 \\ \epsilon\end{array}\right)$

where $\epsilon$ is a complex number modeling the elliptical polarization. Since we assume that birefringence was induced once the beam was linearly polarized, both states of polarization in Eq. (24) are coherent and will therefore create fields that will add up coherently. We calculated the rejection ratio as a function of $\epsilon$ and found similar results than with phase errors for similar values of $\epsilon$ (see Table 3). Since the fields are added coherently, the phase of $\epsilon$ will also affect the rejection ratio. In Table 3, we report the allowed values of $\epsilon$ in order to have a rejection ratio of $10^{5}$ and $10^{6}$. These values of $\epsilon$ correspond to the worst case of phase of $\epsilon$ (when both fields due to longitudinal polarization and due to birefringence are in phase).

Note that $\epsilon$ cannot represent imperfections in the polarizers, since this would induce mixing of incoherent states of polarization, which is not taken into account here. However, the rejection ratio would not be affected if we consider identical imperfections for all polarizers. The effect of non perfectly identical polarizers is a second-order effect not considered in this study. Note also that a similar analysis can be conducted for the orthogonal incoming polarization. This discussion is therefore valid as well with non-polarized incoming beams.

From these results, we can conclude that typical values of $5 \mathrm{mrad}$ or less in phase errors and 0.005 or less in polarization errors would lead to a $10^{5}$ rejection ratio, while $1 \mathrm{mrad}$ or less in phase errors and 0.001 or less in polarization errors is needed to reach a rejection ratio of $10^{6}$.

\section{Polarization-based nulling interferometers}

In the previous sections, we have considered nulling interferometers in which destructive interference is achieved by phase shifting. In this section, we consider nulling interferometers based on rotation of the polarization instead of phase shifting (Spronck et al. 2006). In such a nulling interferometer, all beams are in phase $\left(\phi_{j}=0\right)$ but the direction of the polarization is rotated $\left(\delta_{j}=\delta_{0}+\psi_{j}\right)$ for each beam (see Fig. 9a). Such polarization rotations can be obtained, for instance, with achromatic half-wave plates.

\subsection{Ray tracing}

Similarly to what has been done in Sect. 2, the electric field in the entrance pupil corresponding to the linearly polarized $j$ th beam can be found by replacing $\delta_{j}=\delta_{0}+\psi_{j}$ into Eq. (1),

$\boldsymbol{E}_{\mathrm{ent}, j}=E_{0} \cos \delta_{0} \boldsymbol{e}_{\mathrm{r}}+E_{0} \sin \delta_{0} \boldsymbol{e}_{\psi}$.

A rotation around the azimuthal axis gives the electric field in the exit pupil,

$$
\begin{aligned}
\boldsymbol{E}_{\mathrm{ex}, j}= & E_{0}\left[\cos \delta_{0} \cos \left(\frac{2 \pi}{N}(j-1)\right) \cos \alpha-\sin \delta_{0} \sin \left(\frac{2 \pi}{N}(j-1)\right)\right] \boldsymbol{e}_{x} \\
& +E_{0}\left[\cos \delta_{0} \sin \left(\frac{2 \pi}{N}(j-1)\right) \cos \alpha+\sin \delta_{0} \cos \left(\frac{2 \pi}{N}(j-1)\right)\right] \boldsymbol{e}_{y} \\
& +E_{0} \cos \delta_{0} \sin \alpha \boldsymbol{e}_{z}
\end{aligned}
$$

The electric field at focus is purely longitudinal and simply given by the sum of all individual longitudinal fields,

$E_{z}=E_{0} N \cos \delta_{0} \sin \alpha$.

A calculation of the electric field corresponding to constructive interference is not straightforward since we first need to find for which phase differences between the beams we have a maximal intensity. This can be done by maximizing the on-axis transverse field or, equivalently, by minimizing the on-axis longitudinal field. If we assume that each beam has a different phase $\phi_{j}$, the on-axis longitudinal field is simply given by

$E_{z}=E_{0} \sum_{j=1}^{N} \exp \left(i \phi_{j}\right) \cos \delta_{0} \sin \alpha$.

This longitudinal field can be cancelled by choosing the following phases $\phi_{j}$,

$\phi_{j}=\frac{2 \pi}{N}(j-1)$ 


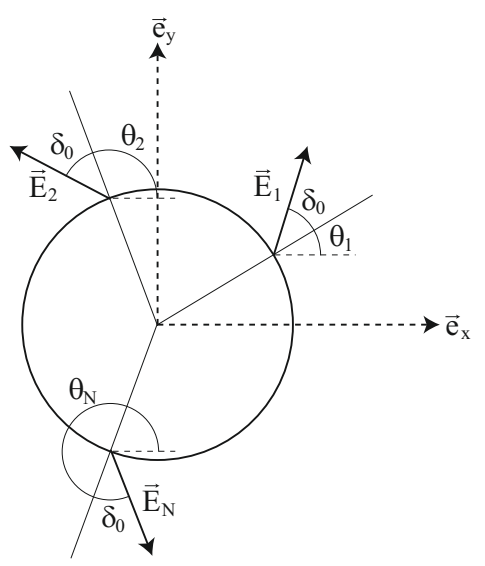

(a)

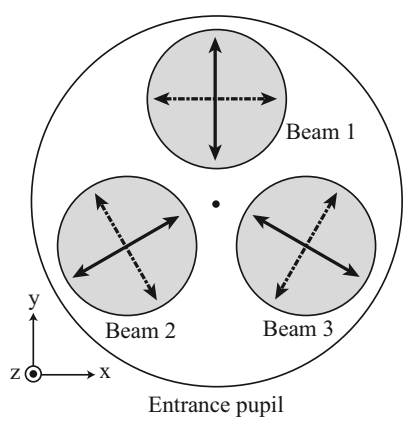

(b)

Fig. 9. Schematic entrance pupil of a multi-axial nulling interferometer based on polarization rotation a) in the case of $N$ ray-like beams and $\mathbf{b}$ ) in the case of three extended beams. The solid arrows represent a quasi-radial polarization while the dotted arrows represent the quasi-azimuthal polarization.

Using these phases, we can calculate the transverse on-axis electric field and thereafter the on-axis electric energy density $\mathcal{U}_{+}$ corresponding to constructive interference. After calculations, we find

$\mathcal{U}_{+}=\left(\cos ^{2} \delta_{0} \cos ^{2} \alpha+\sin ^{2} \delta_{0}\right) \begin{cases}4\left|E_{0}\right|^{2}, & \text { if } N=2, \\ \left|E_{0}\right|^{2} N^{2} / 2, & \text { if } N \neq 2 .\end{cases}$

Comparing Eq. (30) with Eq. (8), we see that both expressions are identical in the case $N=2$, which is normal since a polarization rotation of $\pi$ and a phase shift of $\pi$ are mathematically equivalent. However, for $N>2$, the maximal intensity of such a polarizationbased interferometer is only half the intensity obtained with a phase-based interferometer. The latter is therefore twice as efficient.

The rejection ratio is given by

$R= \begin{cases}\frac{\left(\cos ^{2} \delta_{0} \cos ^{2} \alpha+\sin ^{2} \delta_{0}\right)}{\sin ^{2} \alpha \cos ^{2} \delta}, & \text { if } N=2, \\ \frac{\left(\cos ^{2} \delta_{0} \cos ^{2} \alpha+\sin ^{2} \delta_{0}\right)}{2 \sin ^{2} \alpha \cos ^{2} \delta}, & \text { if } N \neq 2 .\end{cases}$

and is therefore inversely proportional to the square of the effective numerical aperture as it is the case for phase-based nulling interferometers. We can see in Eq. (31) that the rejection ratio is minimal for a quasi-radial polarization distribution $\left(\delta_{0}=0\right)$ and is infinite for quasi-azimuthal polarization distribution $\left(\delta_{0}=\pi / 2\right)$.

Note that in the two-beam case, both phase- and polarization-based interferometers are mathematically equivalent. The quasiradial and quasi-azimuthal polarizations correspond respectively to the cases where the polarization is along the baseline and perpendicular to the baseline.

\subsection{Electric field distribution}

In this section, we will only consider the case of three beams with quasi-azimuthal and quasi-radial polarizations as depicted in Fig. 9 b.

The field distribution in the focal plane for quasi-azimuthal and quasi-radial polarizations are depicted in Figs. 10a and 10b. We can see that, as expected from the ray tracing model, the longitudinal field is equal to zero on-axis in the quasi-azimuthal case. The rejection ratio would therefore be infinite. For quasi-radial polarization, the longitudional field is maximal on-axis, leading to a limited rejection ratio.

We can also show in the case of polarization-based interferometers that the rejection ratio is in very good agreement with Eq. (31). Therefore, we validated with rigorous diffraction theory all results obtained with a simple ray tracing approach.

\subsection{Single-mode fiber filtering for polarization-based nulling interferometers}

In the case of a nulling interferometer based on polarization rotation, we can show (see Appendix B) that the coupling efficiencies to the odd $\left(\xi_{\mathrm{o},-}\right)$ and to the even $\left(\xi_{\mathrm{e},-}\right)$ modes corresponding to destructive interference are both equal to zero,

$\xi_{\mathrm{o},-}=\xi_{\mathrm{e},-}=0$.

For nulling interferometers based on the rotation of polarization, light coming from an on-axis point source is not coupled in the fiber. The rejection ratio will therefore be infinite for any number of beams, any effective numerical aperture and any incoming polarization. Since the coupling efficiencies are rigorously equal to zero for any polarization and any effective numerical aperture 

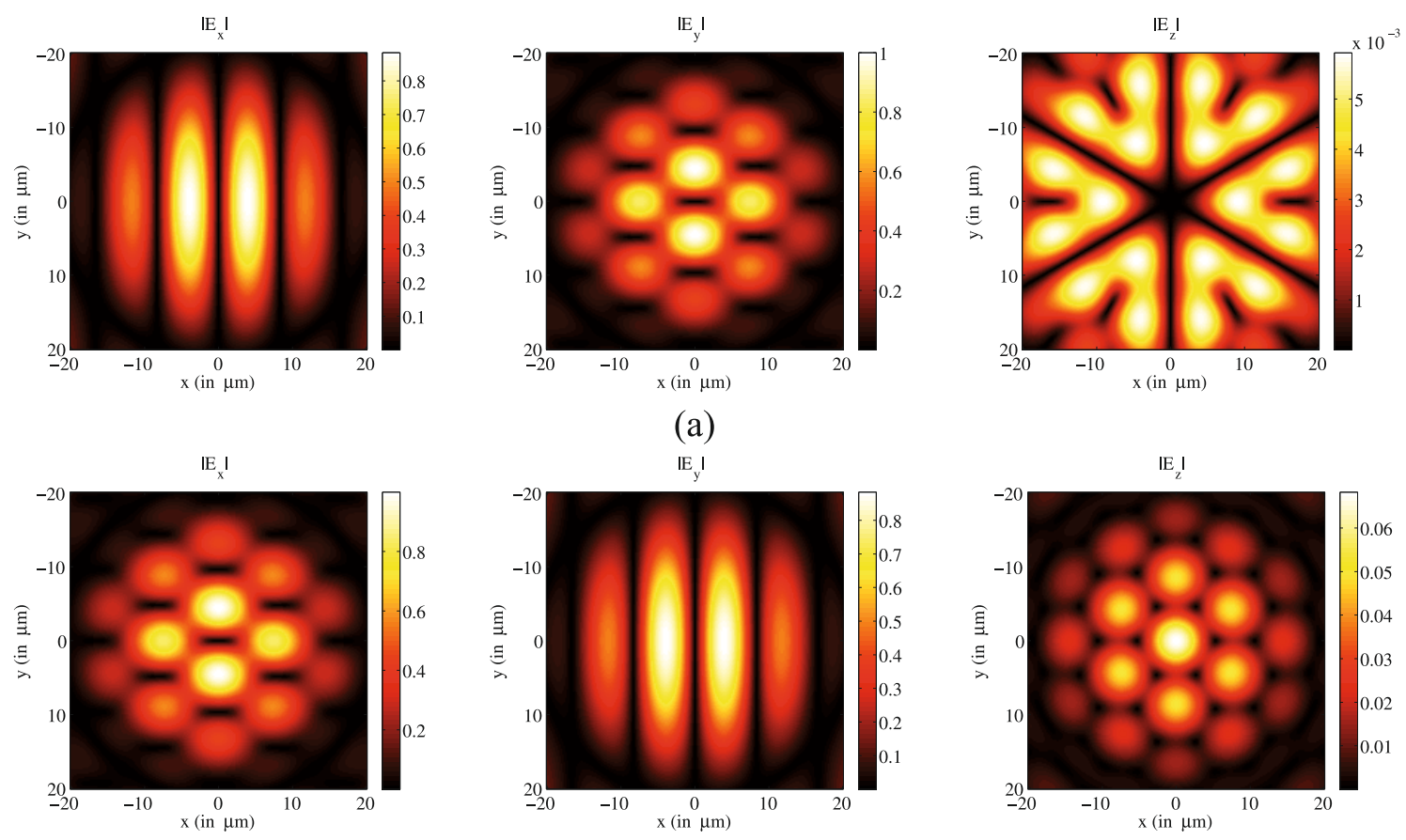

(b)

Fig. 10. Three components of the electric field $\left(\left|E_{x}\right|,\left|E_{y}\right|\right.$ and $\left.\left|E_{z}\right|\right)$ in the focal plane of the focusing optics in the case of a three-beam multiaxial combiner. The beams are in phase and are linearly polarized along either the azimuthal axis a) or the radial axis $\mathbf{b})$. In each case, the three components of the field have been normalized to the largest component.

and by linearity of the coupling efficiencies, we can conclude that the results obtained in this section are also valid in the case of extended beams.

For the coupling efficiencies corresponding to constructive interference, we find after calculations

$\xi_{0,+} \approx-2 \pi K G_{1} I_{1} \begin{cases}2 \sin \delta, & \text { if } N=2, \\ N i / 2 \exp (-i \delta), & \text { if } N \neq 2,\end{cases}$

and

$\xi_{e,+} \approx-2 \pi K G_{1} I_{1} \begin{cases}2 \cos \delta, & \text { if } N=2, \\ N / 2 \exp (-i \delta), & \text { if } N \neq 2 .\end{cases}$

The output power corresponding to constructive interference is then given by

$P_{\text {out }}=4 \pi^{2} K^{2}\left|G_{1} I_{1}\right|^{2} P \begin{cases}4, & \text { if } N=2, \\ N^{2} / 2, & \text { if } N \neq 2 .\end{cases}$

Comparing to phase-based interferometers (Eq. (20)), we see that in the case of two beams, the output power is nearly identical. However, for $N>2$, the efficiency of a polarization-based interferometer is only half the efficiency of a phase-based interferometer. The price to pay to reach an infinite rejection ratio for any number of beams, any effective numerical aperture and any incoming polarization is therefore a loss of half the flux coming from the planet.

\section{Conclusions}

We performed a detailed analysis of multi-axial beam combination for nulling interferometry. We first used a simple ray-tracing model to illustrate the problem. With that model, we have shown that a longitudinal field is created by the focusing optics. This longitudinal component drastically limits the performance of the interferometer. We have shown that the rejection ratio is inversely proportional to the square of the effective numerical aperture and, except in the two-beam case, is independent of the polarization. In the case of a two-beam interferometer, the rejection ratio would be limited if beams were initially linearly polarized along the baseline. If the polarization is perpendicular to the baseline, the rejection ratio is theoretically infinite. We also investigated the case of nulling interferometers based on rotation of the polarization. We have seen that the rejection ratio is also in this case inversely proportional to the square of the effective numerical aperture, is minimal for quasi-radial polarization and infinite for quasi-azimuthal polarization.

We then performed a three-dimensional electric field analysis in the focal plane of a multi-axial nulling interferometer using rigorous diffraction theory. With that model, we analyzed the electric field distribution in the case of two- and three-beam nulling 
interferometers. We have shown that results obtained with rigorous diffraction theory were in very good agreement with the results obtained by ray tracing, therefore confirming these results. In a typical set-up with $\mathrm{NA}_{\mathrm{eff}}=0.06$, the rejection ratio would be of the order of $10^{2}-10^{3}$ if both polarizations are used, which is too low for Earth-like exoplanet detection.

We also have investigated the filtering capabilities of a single-mode optical fiber placed at focus of a multi-axial beam combiner with respect to the longitudinal polarization issue. We have seen that the transverse magnetic fields of the fundamental modes are centro-symmetric functions. As a consequence, any incoming field with anti-symmetric transverse electric fields will not be coupled into the fiber. The fiber is therefore a perfect filter for these anti-symmetric transverse electric fields.

We have given a rigorous analytical expression for the coupling efficiencies and for the rejection ratio in the case of ray-like beams. We have seen that the rejection ratio in a three-beam phase-based nulling interferometer is in good approximation inversely proportional to the fourth power of the effective numerical aperture. For any other number of beams, the theoretical rejection ratio is infinite regardless of the polarization. We have studied the sensitivity of multi-axial nulling interferometers with respect to some imperfections and have shown that typical values of $5 \mathrm{mrad}$ (resp. $1 \mathrm{mrad}$ ) or less in phase errors and 0.005 (resp. 0.001 ) or less in polarization errors were acceptable to reach a $10^{5}$-rejection ratio (resp. $10^{6}$ ). We have also shown that, for the case of polarizationbased nulling interferometers, the rejection ratio would be theoretically infinite for any number of beams, any effective numerical aperture and any incident polarization. However, this infinite rejection ratio occurs at the expense of half the photon flux coming from the planet.

Finally, we have validated these results with numerical simulations in the case of finite-size beams. Except in the three-beam case, the rejection ratio is theoretically infinite for all polarizations. The fiber is therefore an essential component of these multi-axial nulling interferometers since it solves the fundamental problem of the longitudinal polarization which occurs even in the perfect case (aberration-free). As a consequence, we can reasonably think that this problem should not drastically affect the performance of the current four-telescope Darwin/TPF mission architecture. However, if an alternative design involving three beams is considered, light will be coupled into the fiber. The rejection ratio is therefore limited for both polarizations. We further have shown that the rejection ratio is, for small effective numerical apertures, inversely proportional to the fourth power of the effective numerical aperture of the system, which leads to less stringent requirements. Indeed, with a typical $\mathrm{NA}_{\mathrm{eff}}=0.06$, the rejection ratio would be of the order of $10^{6}$ or $10^{8}$ taking the interface into account (note that the interface would lead to a loss of photon flux). In this case, the single-mode fiber does not completely filter out the incoming electric field. However, the amount of light coupled in the fiber in the case of destructive interference is sufficiently low to allow Earth-like exoplanet detection. Therefore, a single-mode fiber is also essential in the three-beam case. The fiber is also an essential component of a polarization-based multi-axial nulling interferometer, since it leads to a theoretically infinite rejection ratio for any number of beams regardless of the polarization.

With this study, we have shown a fundamental limitation of multi-axial beam combiners, which can be solved by means of fiber filtering. We conclude that the longitudinal field component should not prevent direct detection of Earth-like exoplanets.

Acknowledgements. The authors would like to thank Prof. John D. Love from the Australian National University and Prof. Joseph J. M. Braat from Delft University of Technology for some fruitful discussions and suggestions. We further acknowledge the support of TNO Science and Industry, The Netherlands.

\section{Appendix A: Expression for the rejection ratio in a single-mode optical fiber}

Consider an incoming field focused onto an optical fiber. The incoming electric field $\boldsymbol{E}_{\text {inc }}(x, y)$ will excite a certain mode $\boldsymbol{E}_{j}(x, y)$ of the fiber with a certain (complex) strength, namely the complex coupling efficiency $\xi_{j}$. As shown by Snyder \& Love (1983), all modes (bound and radiation modes) of a non-absorbing waveguide satisfy the orthogonality relation

$$
\iint\left(\boldsymbol{E}_{j}(x, y) \times \boldsymbol{H}_{k}^{*}(x, y)\right) \cdot \boldsymbol{z} \mathrm{d} x \mathrm{~d} y=\delta_{j k} \iint\left(\boldsymbol{E}_{j}(x, y) \times \boldsymbol{H}_{j}^{*}(x, y)\right) . z \mathrm{~d} x \mathrm{~d} y,
$$

where $\boldsymbol{H}_{k}^{*}(x, y)$ is the complex conjugate of the magnetic field $\boldsymbol{H}_{k}$ of the $k$ th mode, $\boldsymbol{z}$ is the unit vector along the axis of the fiber and $\delta_{j k}$ is the Kronecker symbol. Using this relation, we can find an expression for the complex coupling efficiency $\xi_{j}$ to the $j$ th mode

$\xi_{j}=\frac{\iint\left(\boldsymbol{E}_{\mathrm{inc}}(x, y) \times \boldsymbol{H}_{j}^{*}(x, y)\right) \cdot \boldsymbol{z} \mathrm{d} x \mathrm{~d} y}{\iint\left(\boldsymbol{E}_{j}(x, y) \times \boldsymbol{H}_{j}^{*}(x, y)\right) \cdot \boldsymbol{z} \mathrm{d} x \mathrm{~d} y}$.

From Eq. (A.2), we can see that the distribution of the longitudinal field does not play any role in the calculation of the coupling efficiencies since only the z-component of the cross product between the incident field and the corresponding modes is used. The electric field $\boldsymbol{E}(x, y)$ propagating inside the fiber is then given by

$\boldsymbol{E}(x, y)=\sum_{j} \xi_{j} \boldsymbol{E}_{j}(x, y)$.

A single-mode fiber has two orthogonal fundamental modes corresponding to the two orthogonal polarizations. In order to keep the same nomenclature as in Snyder \& Love (1983), we will call these modes even $\boldsymbol{E}_{\mathrm{e}}$ and odd $\boldsymbol{E}_{\mathrm{o}}$. An expression for these modes is given in Snyder \& Love (1983). The electric field at the output of the fiber is then given by

$\boldsymbol{E}(x, y)=\boldsymbol{\xi}_{\mathrm{e}} \boldsymbol{E}_{\mathrm{e}}(x, y)+\xi_{\mathrm{o}} \boldsymbol{E}_{\mathrm{o}}(x, y)$,

where $\xi_{\mathrm{e}}$ and $\xi_{\mathrm{o}}$ are given by Eq. (A.2). For the magnetic field, we have

$\boldsymbol{H}(x, y)=\xi_{\mathrm{e}} \boldsymbol{H}_{\mathrm{e}}(x, y)+\xi_{\mathrm{o}} \boldsymbol{H}_{\mathrm{o}}(x, y)$, 
where $\boldsymbol{H}_{\mathrm{e}}$ and $\boldsymbol{H}_{\mathrm{o}}$ denote respectively the magnetic fields of the even and the odd fundamental modes of the fiber. The average energy flow is then given by the Poynting vector $\boldsymbol{S}$ defined as

$\boldsymbol{S}(x, y)=\frac{1}{2} \mathfrak{R}\left[\boldsymbol{E}(x, y) \times \boldsymbol{H}^{*}(x, y)\right] \cdot$

The output power is found by integrating the $z$-component of the Poynting vector $S_{z}(x, y)$ over the $x y$-plane. Since both even and odd modes are orthogonal in the sense of Eq. (A.1) and since the powers corresponding to these modes are equal $\left(P_{\mathrm{e}}=P_{\mathrm{o}}=P\right)$, we find for the output power

$P_{\text {out }}=\left(\left|\xi_{\mathrm{e}}\right|^{2}+\left|\xi_{\mathrm{o}}\right|^{2}\right) P$.

Knowing the electric field distributions in the focal plane of the focusing optics for both constructive and destructive interferences, we can calculate the different coupling efficiencies and therefore the output powers corresponding to constructive $\left(P_{\text {out },+}\right)$ and destructive $\left(P_{\text {out,--}}\right)$ interferences. The rejection ratio after fiber filtering is then given by

$R_{\text {out }}=\frac{P_{\text {out },+}}{P_{\text {out },-}}=\frac{\left|\xi_{\mathrm{e},+}\right|^{2}+\left|\xi_{\mathrm{o},+}\right|^{2}}{\left|\xi_{\mathrm{e},-}\right|^{2}+\left|\xi_{\mathrm{o},-}\right|^{2}}$.

Note that this expression is only valid in the monochromatic case.

To calculate the coupling efficiencies given by Eq. (A.2), we need to know the magnetic fields of the fundamental modes. An expression for these fields in the case of a step-index single-mode fiber is given in Snyder \& Love (1983). The transverse magnetic fields for both even and odd modes can be rewritten

$H_{x, \mathrm{e}}(r, \phi)=A_{2}(r) \sin (2 \phi)$,

$H_{y, \mathrm{e}}(r, \phi)=-A_{1}(r)-A_{2}(r) \cos (2 \phi)$,

$H_{x, 0}(r, \phi)=A_{1}(r)-A_{2}(r) \cos (2 \phi)$,

$H_{y, 0}(r, \phi)=-A_{2}(r) \sin (2 \phi)$,

where

$$
\begin{gathered}
A_{1}(r)=\left(\frac{\epsilon_{0}}{\mu_{0}}\right)^{1 / 2} \frac{k n_{c a}^{2}}{\beta}\left\{\begin{array}{ll}
a_{3} \frac{J_{0}(U r / a)}{J_{1}(U)}, & \text { if } r \leq a \\
\frac{U}{W} a_{5} \frac{K_{0}(W a)}{K_{1}(W)}, & \text { if } r>a
\end{array},\right. \\
A_{2}(r)=\left(\frac{\epsilon_{0}}{\mu_{0}}\right)^{1 / 2} \frac{k n_{c o}^{2}}{\beta}\left\{\begin{array}{ll}
a_{4} \frac{J_{2}(U r / a)}{J_{1}(U)}, & \text { if } r \leq a \\
-\frac{U}{W} a_{6} \frac{K_{2}(W r / a)}{K_{1}(W)}, & \text { if } r>a
\end{array} .\right.
\end{gathered}
$$

We can see from Eq. (A.9) that all even and odd transverse magnetic fields are centro-symmetric functions $\left(H_{e, t}(r, \phi)=H_{e, t}(r, \pi+\phi)\right.$ and $\left.H_{o, t}(r, \phi)=H_{o, t}(r, \pi+\phi)\right)$. Therefore, if the incoming electric field has anti-symmetric transverse components, both coupling efficiencies in Eq. (A.2) will be equal to zero: such a field will not be coupled into the fiber.

\section{Appendix B: Calculation of the coupling efficiencies}

In this section, we will derive the expressions for the coupling efficiencies corresponding to destructive and constructive interferences in the case of phase and polarization-based interferometers.

The coupling efficiency $\xi_{0,-}$ to the odd mode is given by

$\xi_{\mathrm{o},-}=\int_{0}^{\infty} \int_{0}^{2 \pi}\left[E_{x}(r, \phi) H_{y, 0}(r, \phi)-E_{y}(r, \phi) H_{x, 0}(r, \phi)\right] r \mathrm{~d} r \mathrm{~d} \phi$.

\section{B.1. Phase-based nulling interferometers}

Consider $N$ ray-like beams regularly spaced on a circle. The position of the $j$ th beam in polar coordinates is given by $\left(k_{\mathrm{r}}, k_{\phi, j}=\frac{2 \pi}{N}(j-1)\right), j=1, . ., N$. The beams are linearly-polarized at an angle $\delta$ and, in order to get on-axis destructive interference, the phase of the $j$ th beam is chosen to be $\phi_{j}=\frac{2 \pi}{N}(j-1)$. Under these conditions, we can use Eq. (12) to find an analytical expression for the transverse electric field distribution in the focal plane. After elementary manipulations, we find

$$
\begin{aligned}
& E_{x}=K \sum_{j=1}^{N}\left\{\left[G_{1} \cos \delta-G_{2} \cos \left(\delta-2 k_{\phi, j}\right)\right] \exp \left(i \phi_{j}\right) \exp \left[i r k_{\mathrm{r}} \cos \left(k_{\phi, j}-\phi\right)\right]\right\}, \\
& E_{y}=K \sum_{j=1}^{N}\left\{\left[G_{1} \sin \delta+G_{2} \sin \left(\delta-2 k_{\phi, j}\right)\right] \exp \left(i \phi_{j}\right) \exp \left[i r k_{\mathrm{r}} \cos \left(k_{\phi, j}-\phi\right)\right]\right\},
\end{aligned}
$$


where

$$
\begin{gathered}
K=-\frac{i \rho}{2 \pi} \frac{\exp \left(i k_{z}\right)}{\sqrt{k_{z} k}} k_{\mathrm{r}}, \\
G_{1}=1 / 2\left(1+k_{z} / k\right), \\
G_{2}=1 / 2\left(1-k_{z} / k\right) .
\end{gathered}
$$

After replacing Eqs. (B.2) and (A.9) into Eq. (B.1) and after putting similar terms together, we find

$$
\begin{aligned}
\xi_{\mathrm{o},-}= & -K \int_{0}^{\infty} \int_{0}^{2 \pi} \sum_{j=1}^{N} G_{1} \sin \delta \exp \left(i \phi_{j}\right) \exp \left[i r k_{\mathrm{r}} \cos \left(k_{\phi, j}-\phi\right)\right] A_{1}(r) r \mathrm{~d} r \mathrm{~d} \phi \\
& -K \int_{0}^{\infty} \int_{0}^{2 \pi} \sum_{j=1}^{N} G_{2} \sin \left(\delta-2 k_{\phi, j}\right) \exp \left(i \phi_{j}\right) \exp \left[i r k_{\mathrm{r}} \cos \left(k_{\phi, j}-\phi\right)\right] A_{1}(r) r \mathrm{~d} r \mathrm{~d} \phi \\
& +K \int_{0}^{\infty} \int_{0}^{2 \pi} \sum_{j=1}^{N} G_{1} \sin (\delta-2 \phi) \exp \left(i \phi_{j}\right) \exp \left[i r k_{\mathrm{r}} \cos \left(k_{\phi, j}-\phi\right)\right] A_{2}(r) r \mathrm{~d} r \mathrm{~d} \phi \\
& +K \int_{0}^{\infty} \int_{0}^{2 \pi} \sum_{j=1}^{N} G_{2} \sin \left(\delta-2 k_{\phi, j}+2 \phi\right) \exp \left(i \phi_{j}\right) \exp \left[i r k_{\mathrm{r}} \cos \left(k_{\phi, j}-\phi\right)\right] A_{2}(r) r \mathrm{~d} r \mathrm{~d} \phi .
\end{aligned}
$$

Using some integral definitions of the Bessel functions (Abramowitz \& Stegun 1968), we find

$$
\begin{aligned}
\xi_{\mathrm{o},-}= & -2 \pi K G_{1} \sin \delta \sum_{j=1}^{N} \exp \left(i \phi_{j}\right) \int_{0}^{\infty} J_{0}\left(r k_{\mathrm{r}}\right) A_{1}(r) r \mathrm{~d} r \\
& -2 \pi K G_{2} \sum_{j=1}^{N} \sin \left(\delta-2 k_{\phi, j}\right) \exp \left(i \phi_{j}\right) \int_{0}^{\infty} J_{0}\left(r k_{\mathrm{r}}\right) A_{1}(r) r \mathrm{~d} r \\
& -2 \pi K G_{1} \sum_{j=1}^{N} \sin \left(\delta-2 k_{\phi, j}\right) \exp \left(i \phi_{j}\right) \int_{0}^{\infty} J_{2}\left(r k_{\mathrm{r}}\right) A_{2}(r) r \mathrm{~d} r \\
& -2 \pi K G_{2} \sum_{j=1}^{N} \sin \left(\delta-2 k_{\phi, j}+2 k_{\phi, j}\right) \exp \left(i \phi_{j}\right) \int_{0}^{\infty} J_{2}\left(r k_{\mathrm{r}}\right) A_{2}(r) r \mathrm{~d} r,
\end{aligned}
$$

where $J_{0}$ and $J_{2}$ denote respectively the zeroth and the second order Bessel functions of the first kind. Finally, after some elementary simplifications, we have

$\xi_{\mathrm{o},-}=\left\{\begin{array}{ll}-3 i \pi K \exp (-i \delta)\left(G_{1} I_{2}+G_{2} I_{1}\right), & \text { if } N=3 \\ 0, & \text { if } N \neq 3\end{array}\right.$,

where $I_{1}=\int_{0}^{\infty} J_{0}\left(r k_{\mathrm{r}}\right) A_{1}(r) r \mathrm{~d} r$ and $I_{2}=\int_{0}^{\infty} J_{2}\left(r k_{\mathrm{r}}\right) A_{2}(r) r \mathrm{~d} r$.

Similarly, for the coupling efficiency corresponding to the even mode $\xi_{\mathrm{e},-}$, we have

$$
\xi_{\mathrm{e},-}=\left\{\begin{array}{ll}
3 \pi K \exp (-i \delta)\left(G_{1} I_{2}+G_{2} I_{1}\right), & \text { if } N=3 \\
0, & \text { if } N \neq 3
\end{array} .\right.
$$

\section{B.2. Polarization-based nulling interferometers}

In the case of a nulling interferometer based on polarization rotation, the transverse components of the electric field in the focal plane are given by

$$
\begin{aligned}
& E_{x}=K \sum_{j=1}^{N}\left\{\left[G_{1} \cos \left(\delta+k_{\phi, j}\right)-G_{2} \cos \left(\delta-k_{\phi, j}\right)\right] \exp \left[i r k_{\mathrm{r}} \cos \left(k_{\phi, j}-\phi\right)\right]\right\}, \\
& E_{y}=K \sum_{j=1}^{N}\left\{\left[G_{1} \sin \left(\delta+k_{\phi, j}\right)+G_{2} \sin \left(\delta-k_{\phi, j}\right)\right] \exp \left[i r k_{\mathrm{r}} \cos \left(k_{\phi, j}-\phi\right)\right]\right\}
\end{aligned}
$$


The coupling efficiencies to the orthogonal fiber modes are given by

$$
\begin{aligned}
\xi_{\mathrm{o},-}= & -2 \pi K G_{1} \sum_{j=1}^{N} \sin \left(\delta+k_{\phi, j}\right) \int_{0}^{\infty} J_{0}\left(r k_{\mathrm{r}}\right) A_{1}(r) r \mathrm{~d} r-2 \pi K G_{2} \sum_{j=1}^{N} \sin \left(\delta-k_{\phi, j}\right) \int_{0}^{\infty} J_{0}\left(r k_{\mathrm{r}}\right) A_{1}(r) r \mathrm{~d} r \\
& -2 \pi K G_{1} \sum_{j=1}^{N} \sin \left(\delta-k_{\phi, j}\right) \int_{0}^{\infty} J_{2}\left(r k_{\mathrm{r}}\right) A_{2}(r) r \mathrm{~d} r-2 \pi K G_{2} \sum_{j=1}^{N} \sin \left(\delta+k_{\phi, j}\right) \int_{0}^{\infty} J_{2}\left(r k_{\mathrm{r}}\right) A_{2}(r) r \mathrm{~d} r
\end{aligned}
$$

and

$$
\begin{aligned}
\xi_{\mathrm{e},-}= & -2 \pi K G_{1} \sum_{j=1}^{N} \cos \left(\delta+k_{\phi, j}\right) \int_{0}^{\infty} J_{0}\left(r k_{\mathrm{r}}\right) A_{1}(r) r \mathrm{~d} r+2 \pi K G_{2} \sum_{j=1}^{N} \cos \left(\delta-k_{\phi, j}\right) \int_{0}^{\infty} J_{0}\left(r k_{\mathrm{r}}\right) A_{1}(r) r \mathrm{~d} r \\
& +2 \pi K G_{1} \sum_{j=1}^{N} \sin \left(\delta-k_{\phi, j}\right) \int_{0}^{\infty} J_{2}\left(r k_{\mathrm{r}}\right) A_{2}(r) r \mathrm{~d} r-2 \pi K G_{2} \sum_{j=1}^{N} \sin \left(\delta+k_{\phi, j}\right) \int_{0}^{\infty} J_{2}\left(r k_{\mathrm{r}}\right) A_{2}(r) r \mathrm{~d} r
\end{aligned} .
$$

Since $\sum_{j=1}^{N} \cos \left(k_{\phi, j}\right)=\sum_{j=1}^{N} \sin \left(k_{\phi, j}\right)=0$, both coupling efficiencies are equal to zero,

$\xi_{\mathrm{o},-}=\xi_{\mathrm{e},-}=0$.

\section{References}

Abramowitz, M., \& Stegun, I. 1968, Handbook of mathematical functions (New York: Dover)

Angel, J. R., Cheng, A. Y. S., \& Woolf, N. J. 1986, Nature, 232, 341

Beichman, C. A., Woolf, N. J., \& Lindensmith, C. A. 1999, The Terrestrial Planet Finder (TPF): a NASA Origins Program to search for habitable planets (Jet Propulsion Laboratory)

Bracewell, R. N. 1978, Nature, 274, 780

Buisset, C., Rejeaunier, X., Rabbia, Y., et al. 2006, in Advances in Stellar Interferometry, Proc. SPIE, 6268, 626819

Fridlund, C. V. M. 2000, in Darwin and Astronomy: the Infrared Space Interferometer, ed. B. Schürmann, ESA SP, 451, 11

Gori, P.-M., van der Avoort, C., Poole, R. S. L., \& Brug, H. V. 2004, in New Frontiers in Stellar Interferometry, Proc. SPIE, 5491, 1011

Haguenauer, P., \& Serabyn, E. 2006, Appl. Opt., 45, 2749

Hao, B., \& Leger, J. 2007, Opt. Express, 15, 3550

Houizot, P., Boussard-Plédel, C., Faber, A. J., et al. 2007, Opt. Express, 15, 12529

Ignatowsky, V. 1919, Tr. Opt. Inst. Petrograd, 1, 1

Lawson, P. R., Lay, O. P., Martin, S. R., et al. 2008, in Optical and Infrared Interferometry, ed. M. Schöller, W. C. Danchi, \& F. Delplancke, Proc. SPIE, 7013, $70132 \mathrm{~N}$

Lay, O. P., Martin, S. R., \& Hunyadi, S. L. 2007, in Techniques and Instrumentation for Detection of Exoplanets III, ed. D. R. Coulter, Proc. SPIE, 6693, 66930A Mayor, M., \& Queloz, D. 1995, Nature, 378, 355

Mennesson, B., Ollivier, M., \& Ruilier, C. 2002, J. Opt. Soc. Am. A, 19, 596

Novotny, L., Beversluis, M. R., Youngworth, K. S., \& Brown, T. G. 2001, Phys. Rev. Lett., 86, 5251

Quabis, S., Dorn, R., Eberler, M., Glöckl, O., \& Leuchs, G. 2001, Appl. Phys. B, 72, 109

Richards, B., \& Wolf, E. 1959, Proc. Roy. Soc. A, 253, 358

Rurimo, G. K., Schardt, M., Quabis, S., et al. 2006, J. Appl. Phys., 100, 023112

Serabyn, E., \& Colavita, M. M. 2001, Appl. Opt., 40, 1668

Snyder, A., \& Love, J. 1983, Optical waveguide theory (London: Chapman and Hall)

Spronck, J., Pereira, S., \& Braat, J. 2006, Opt. Express, 14, 2657

van de Nes, A. S., Billy, L., Pereira, S. F., \& Braat, J. J. M. 2004, Opt. Express, 12, 1281

Wallner, O., \& Leeb, W. 2002, J. Opt. Soc. Am. A, 19, 2445

Wallner, O., Leeb, W., \& Flatscher, R. 2003, in Interferometry for Optical Astronomy II, Proc. SPIE, 4838, 668

Wallner, O., Armengol, J. P., \& Karlsson, A. 2004, in New Frontiers in Stellar Interferometry, Proc. SPIE, 5491, 1417 